\title{
Association between previously identified loci affecting telomere length and coronary heart disease (CHD) in Han Chinese population
}

This article was published in the following Dove Press journal:

Clinical Interventions in Aging

27 May 2014

Number of times this article has been viewed

\section{Hui Ding' \\ Fen Yan' \\ Lin-Lin Zhou ${ }^{2}$ \\ Xiu-Hai Ji ${ }^{3}$ \\ Xin-Nan Gu' \\ Zhi-Wei Tang' \\ Ru-Hua Chen'}

'Department of Pulmonary Medicine, The Affiliated Yixing People's Hospital, Jiangsu University, Zhenjiang, Jiangsu

Province, ${ }^{2}$ Department of Cardiology, Affiliated Cixi Hospital, Wenzhou Medical University, Wenzhou, Zhejiang Province, ${ }^{3}$ Department of Oncology, Affiliated Taicang Hospital of Traditional Chinese Medicine, Suzhou, Jiangsu Province, People's Republic of China
Correspondence: Ru-Hua Chen Department of Pulmonary Medicine, The Affiliated Yixing People's Hospital, Jiangsu University, 30I Xuefu Road, Zhenjiang 212013, Jiangsu Province, People's Republic of China

Tel +86 I3706I5 6506

Fax +86 510 8792 III0

Emaildh I3505।@I63.com
Purpose: To replicate previously confirmed telomere-length loci in a Chinese Han population with coronary heart disease (CHD), and investigate these loci and the possibility of and age at onset of CHD.

Patients and methods: 1514 CHD patients and 2470 normal controls were recruited. Medical data including age, sex, body mass index, lipid profiles, history of hypertension, type 2 diabetes mellitus, and dyslipidemia were collected from all the participants. Seven previously identified single-nucleotide polymorphisms (SNPs) related to leucocyte telomere length were genotyped, including rs 10936599 in TERC, rs2736100 in TERT, rs7675998 in NAF1, rs9420907 in OBFC1, rs8105767 in ZNF208, rs755017 in RTEL1, and rs11125529 in ACYP2.

Results: No significant difference in genotype frequencies from the Hardy-Weinberg equilibrium test was noted for all tested SNPs both in the CHD patients and the normal controls. No polymorphism was observed for rs9420907, and AA genotype was noted in both the CHD patients and the controls. Neither the genotype nor the allele frequencies of rs 2736100 , rs8105767, rs11125529, and rs2967374 were significantly different between the CHD patients and the normal controls. For rs10936599 and rs755017, statistical difference was found for the allele frequency but not genotype. Distributions of genotype and allele were significantly different between the two groups for rs 7675998. The odds ratio for carriers of CHD was 2.127 (95\% confidence interval: 1.909-2.370) for the A allele of rs7675998. By one-way analysis of variance test, rs7675998 was associated with the onset age of CHD. CHD patients with the AA genotype of rs7675998 had significantly lower onset age $(P<0.05)$.

Conclusion: In a Chinese Han population, $N A F 1$ gene encoding proteins with known function in telomere biology may influence both the possibility of and the age at onset of CHD, as previously reported in European studies.

Keywords: coronary heart disease, gene, leucocyte telomere length

\section{Introduction}

Coronary heart disease (CHD), synonymously called coronary artery disease (CAD) is the most predominant among the cardiovascular diseases. Multiple factors, including genetic, environmental, and psychological factors, were believed to contribute to the onset of CHD. ${ }^{1}$ The majority of CHD cases could be explained by the interaction of genetic and environmental factors. ${ }^{2,3}$ However, at an individual level there is wide variation in both the occurrence of CHD and the age of manifestation, even in individuals with the same risk factor profiles. Given that CHD is an age-related disease, the hypothesis has been proposed that inter-individual variation in risk of CHD might come from variation in the rate of biological aging. ${ }^{46}$ Telomeres are the protein-bound DNA repeat structures at the ends of chromosomes that play an important role in maintaining 
genomic stability. They are critical in regulating cellular replicative capacity, and are always deemed as a marker of biological aging. ${ }^{7-10}$ In a large, cross-sectional study, mean leucocyte telomere length (LTL) was found to be a predictor of future CHD events in middle-aged, high-risk men and might be able to identify individuals who would benefit most from statin treatment. ${ }^{4}$ This supports the hypothesis that differences in biological aging might contribute to the risk and variability in age of the onset of CHD. ${ }^{5,6} \mathrm{~A}$ recent genomewide meta-analysis identified seven loci involved in telomere biology, including rs 10936599 in TERC, rs2736100 in TERT, rs7675998 in NAF1, rs9420907 in OBFC1, rs8105767 in ZNF208, rs755017 in RTEL1, and rs11125529 in ACYP2, and the subsequent large, case-control study confirmed the association between shorter LTL and increased risk of coronary artery disease (21\% [95\% confidence interval, $5 \%-35 \%$ ] per standard deviation in LTL, $P=0.014$ ) in those of European descent. ${ }^{11}$ The aim of this study is to replicate these previously confirmed telomere-length loci in a Chinese Han population with CHD.

\section{Materials and methods Study subjects}

1514 CHD patients and 2470 normal controls were recruited from a single province (Jiangsu Province) in the People's Republic of China. CHD was diagnosed by angiography, which was defined as the presence of at least one significant coronary artery stenosis of $\geq 50 \%$ luminal diameter on coronary angiography. For normal controls, the criteria were no history of CAD or electrocardiographic signs of CAD. All participants were Han ethnic. Patients with myocardial bridge, congenital heart diseases, or other types of atherosclerotic lesions were excluded from the study. The individuals in the CHD and control groups were all unrelated, and identity information of all the subjects has been critically reviewed.

\section{Data and sample collections}

Medical data including age, sex, body mass index (BMI), lipid profiles, history of hypertension, type 2 diabetes mellitus, and dyslipidemia were collected from all the participants. The BMI was calculated as the weight $(\mathrm{kg})$ divided by the corrected height $(\mathrm{m})$ squared $\left(\mathrm{kg} / \mathrm{m}^{2}\right)$. Rest electrocardiograms (ECG) were performed for the control participants. All control participants showed no signs of CHD, hypertension, diabetes mellitus, or dyslipidemia based on the ECG results and medical-case files at the time of enrollment. Total cholesterol (TC), total triglyceride (TG), high density lipoprotein-cholesterol (HDL-C), low density lipoprotein-cholesterol (LDL-C), apolipoprotein (apo)AI, apoB, lipoprotein ( $\mathrm{Lp}[\mathrm{a}]$ ), and $\mathrm{C}$ reactive protein $(\mathrm{CRP})$ were measured by standard techniques.

\section{Selection of single-nucleotide polymorphisms}

Seven previously identified single-nucleotide polymorphisms (SNPs) related to LTL were genotyped, including rs10936599 in TERC, rs2736100 in TERT, rs7675998 in NAF1, rs9420907 in OBFC1, rs8105767 in ZNF208, rs755017 in RTEL1, and rs11125529 in ACYP2.

\section{Genotyping}

Approved by the ethics committees of The Affiliated Yixing People's Hospital, Jiangsu University, blood samples were collected for DNA analysis, with informed consent obtained from the participants. Standard protocols were used to extract genomic DNA from blood leukocytes. Genotyping was performed using polymerase chain reaction (PCR)based Invader assay with the probe sets designed and synthesized by Third Wave (Shanghai, People's Republic of China). The genotyping results were read with an ABI PRISM7900HT sequence detection system (Applied Biosystems, Foster City, CA, USA). Twenty percent of the samples were selected randomly to validate the reproducibility of the genotyping results, with $100 \%$ reproducibility. Two authors independently reviewed the genotyping results, data entry, and statistical analysis.

\section{Statistical analysis}

Data were analyzed using SPSS software (SPSS Inc., Chicago, IL, USA, version 13.0). Data are expressed as the mean \pm standard deviation (SD). The significance of differences was evaluated using the Student's $t$-test for continuous variables and the $\chi^{2}$ test for non-continuous variables. The $\chi^{2}$ test was used to examine differences of allelic frequencies and genotype distributions between the CHD patients and the controls. The Hardy-Weinberg equilibrium was tested by a goodness-of-fit $\chi^{2}$ test. Odds ratios (ORs) of the significant associated SNPs were calculated using the non-risk allele as a reference. Multivariate analysis was performed using a logistic regression analysis for independent variables that were related to the presence or absence of CHD. A one-way analysis of variance (ANOVA) test was used to compare the mean onset age of different genotypes in case-only analyses. Statistical significance was set at $P<0.05$. 


\section{Results}

\section{Characteristics of study subjects}

Clinical characteristics of the CHD patients and control subjects are shown in Table 1. The proportion of males, average age, and BMI were all significantly higher in the CHD patients than in the controls. The ratios of hypertension, diabetes, and hyperlipidemia were higher in the CHD patients than in the controls. Plasma concentrations of LDL-C and HDL-C were significantly lower, while TC, TG, and Lp(a) were significantly higher in the CHD group compared to the control group. No differences in ApoA1 and ApoB were found between the two groups.

\section{Case-control association analysis}

No significant difference in genotype frequencies from the Hardy-Weinberg equilibrium test was noted for any tested SNPs, both in the CHD patients and the normal controls. No polymorphism was observed for rs 9420907 , and the AA genotype was noted in both the CHD patients and the controls. Neither the genotype nor the allele frequencies of rs2736100, rs8105767, rs11125529, and rs2967374 were significantly different between the CHD patients and the normal controls (Table 2). For rs10936599 and rs755017, a statistically significant difference was found for the allele frequency but not the genotype. Distributions of genotype and allele were significantly different between the two groups for rs 7675998. The ORs for carriers for CHD of each allele are listed in Table 3.

Table I The characteristics of the clinical data in comparisons between control and CHD groups

\begin{tabular}{|c|c|c|c|}
\hline & $\begin{array}{l}\text { CHD patients } \\
(n=I, 5 \mid 4)\end{array}$ & $\begin{array}{l}\text { Controls } \\
(n=2,470)\end{array}$ & $P$-value \\
\hline Age (years) & $63.7 \pm 9.5$ & $58.6 \pm 8.9$ & 0.002 \\
\hline Sex (male/female) & $977 / 537$ & $\mathrm{I}, 562 / 908$ & $0.4 I$ \\
\hline BMI $\left(\mathrm{kg} / \mathrm{m}^{2}\right)$ & 25.8 & 24.2 & $<0.01$ \\
\hline Smoking (\%) & 52.1 & 34.3 & $<0.01$ \\
\hline DM (\%) & 58.4 & 42.3 & $<0.01$ \\
\hline Hypertension (\%) & 25.6 & 10.2 & $<0.01$ \\
\hline TC (mmol/L) & $5.42 \pm 1.03$ & $4.92 \pm 0.92$ & $<0.01$ \\
\hline TG (mmol/L) & $1.96 \pm 0.72$ & $1.38 \pm 0.43$ & $<0.01$ \\
\hline LDL-C (mmol/L) & $3.40 \pm 0.54$ & $3.02 \pm 0.32$ & $<0.01$ \\
\hline HDL-C (mmol/L) & $1.22 \pm 0.8 \mathrm{I}$ & $|.47 \pm 0.5|$ & $<0.01$ \\
\hline ApoAI (g/L) & $1.32 \pm 0.14$ & $1.30 \pm 0.13$ & 0.26 \\
\hline ApoB (g/L) & $0.91 \pm 0.21$ & $0.90 \pm 0.16$ & 0.52 \\
\hline Lp (a) (mg/L) & $292.48 \pm 226.83$ & $43.27 \pm 72.63$ & $<0.01$ \\
\hline
\end{tabular}

Abbreviations: CHD, coronary heart disease; BMI, body mass index DM, diabetes mellitus; TC, total cholesterol; TG, total triglyceride; HDL-C, high density lipoprotein-cholesterol; LDL-C, low density lipoprotein-cholesterol; Apo, apolipoprotein; $L p(a)$, lipoprotein.

\section{Case-only association study}

By one-way ANOVA test, rs7675998 was associated with the onset age of CHD. CHD patients with the AA genotype of rs7675998 had significantly lower age of onset $(P<0.05$; Table 4).

\section{Discussion}

Telomeres are the extreme ends of chromosomal DNA, playing a critical role in maintaining genomic stability by regulating cellular replicative capacity. ${ }^{12-14}$ Due to the inability of DNA polymerase to fully complete the replication of the 3' end of linear DNA, telomeres progressively shorten with repeated cell division. ${ }^{13}$ Once a critically short telomere length is reached, the cell is triggered to enter replicative senescence, which subsequently leads to cell death. ${ }^{14}$ Therefore, mean telomere length could serve as a marker for biological age, at least at the cellular level, with shorter telomeres indicating increased biological age. ${ }^{10,15} \mathrm{CHD}$ is an age-related disease and many studies have confirmed the consolidate association between leukocyte telomere length and CHD. ${ }^{4-6} \mathrm{CHD}$ is a complex, multifactorial disorder involving multiple environmental risks and genetic factors, and LTL was found to be related to many of these factors. ${ }^{1} \mathrm{CHD}$ is a chronic inflammatory process and the shorter telomere length in individuals prone to CHD could simply indicate a greater white blood cell turnover. ${ }^{16-18}$ Increased oxidative stress was another factor contributing to atherosclerosis, and increased oxidant stress has been shown to increase rates of telomere attrition in vitro. ${ }^{19-21}$ Other risk factors of CHD, including smoking, high BMI, and type 1 diabetes mellitus, were also associated with shorter LTL. ${ }^{22-24}$ As to psychological aspects, life stress and job insecurity have been implicated in the etiology of CHD. ${ }^{3,25}$ Recent studies have demonstrated a strong correlation between life stress and shorter LTL. ${ }^{26}$ In a word, shorter LTL may pose cumulative effects on pathogenesis of CHD.

LTL is significantly variable among individuals, and was thought to be determined genetically. ${ }^{11}$ It is believed that those individuals who have inherited and are born with shorter telomeres might be more prone to some certain diseases. Two loci on chromosome 3q26 (TERC) and chromosome 10q24.33 (OBFC1) have shown an association with mean LTL in a genome-wide association study (GWAS). ${ }^{11}$ In a subsequent GWAS with more cases, five new loci were also associated with LTL, and showed an association with increased risks of CHD. ${ }^{11}$ From the viewpoint of population genetics, generalization of LTL association results previously 
Table 2 Distribution of selected variables among cases and controls in a Han Chinese population

\begin{tabular}{|c|c|c|c|c|c|c|c|c|c|c|c|}
\hline \multirow[t]{2}{*}{ Genotype } & \multicolumn{2}{|l|}{ CHD } & \multicolumn{2}{|c|}{ Control } & \multirow[t]{2}{*}{$\chi^{2}(P)$} & \multirow[t]{2}{*}{ Allele } & \multicolumn{2}{|l|}{ CHD } & \multicolumn{2}{|c|}{ Control } & \multirow[t]{2}{*}{$\chi^{2}(P)$} \\
\hline & $\mathbf{n}$ & $\%$ & $\mathbf{n}$ & $\%$ & & & $\mathbf{n}$ & $\%$ & $\mathbf{n}$ & $\%$ & \\
\hline \multicolumn{12}{|l|}{ rs 10936599} \\
\hline $\mathrm{CC}$ & 265 & 17.5 & 371 & 15.0 & 0.08 & C & I,298 & 42.9 & 2,001 & 40.5 & 0.04 \\
\hline $\mathrm{CT}$ & 768 & 50.7 & 1,259 & 51.0 & & $\mathrm{~T}$ & I,730 & 57.1 & 2,939 & 59.5 & \\
\hline $\mathrm{TT}$ & 481 & 31.8 & 840 & 34.0 & & & & & & & \\
\hline \multicolumn{12}{|l|}{ rs2736100 } \\
\hline GG & 357 & 23.6 & 599 & 24.3 & 0.56 & G & 1,499 & 49.5 & 2,436 & 49.3 & 0.87 \\
\hline GT & 785 & 51.8 & 1,238 & 50.1 & & $\mathrm{~T}$ & I,529 & 50.5 & 2,504 & 50.7 & \\
\hline TT & 372 & 24.6 & 633 & 25.6 & & & & & & & \\
\hline \multicolumn{12}{|l|}{ rs7675998 } \\
\hline $\mathrm{AA}$ & 145 & 9.6 & 50 & 2.0 & 0.000 & $A$ & 889 & 29.3 & 815 & 16.5 & 0.000 \\
\hline AG & 599 & 39.6 & 715 & 28.9 & & G & 2,139 & 70.7 & 4,125 & 83.5 & \\
\hline GG & 770 & 50.9 & I,705 & 69.1 & & & & & & & \\
\hline \multicolumn{12}{|l|}{ rs9420907 } \\
\hline $\mathrm{AA}$ & $1,5 \mid 4$ & 100 & 2,470 & 100 & N/A & A & 3,028 & 100 & 4,940 & 100 & $\mathrm{~N} / \mathrm{A}$ \\
\hline$A C$ & & & & & & C & & & & & \\
\hline \multicolumn{12}{|l|}{$\mathrm{CC}$} \\
\hline \multicolumn{12}{|l|}{ rs8105767 } \\
\hline $\mathrm{AA}$ & 730 & 48.2 & 1,223 & 49.5 & 0.73 & $A$ & 2,124 & 71.1 & 3,502 & 70.9 & 0.48 \\
\hline AG & 664 & 43.9 & I,056 & 42.8 & & G & 904 & 29.9 & $\mathrm{I}, 438$ & 29.1 & \\
\hline GG & 120 & 7.9 & 191 & 7.7 & & & & & & & \\
\hline \multicolumn{12}{|l|}{ rs7550I7 } \\
\hline $\mathrm{AA}$ & 485 & 32.0 & 672 & 27.2 & 0.41 & $A$ & 1,736 & 57.3 & 2,598 & 52.6 & 0.000 \\
\hline$A G$ & 766 & 50.6 & $\mathrm{I}, 254$ & 50.8 & & G & 1,292 & 42.7 & 2,342 & 47.4 & \\
\hline GG & 263 & 17.4 & 544 & 22.0 & & & & & & & \\
\hline \multicolumn{12}{|l|}{ rsIII 25529} \\
\hline AA & 254 & 16.8 & 453 & 18.3 & 0.42 & $A$ & I,305 & 43.1 & 2,168 & 43.9 & 0.49 \\
\hline$A C$ & 797 & 52.6 & 1,262 & 51.1 & & $C$ & I,723 & 56.9 & 2,772 & 56.1 & \\
\hline $\mathrm{CC}$ & 463 & 30.6 & 755 & 30.6 & & & & & & & \\
\hline
\end{tabular}

Abbreviations: $\mathrm{CHD}$, coronary heart disease; N/A, not available.

identified in European GWA studies (or meta-analyses) to non-European populations is an interesting issue, because it can facilitate the fine mapping of common causal variants by providing clues as to whether SNPs identified in European GWA studies are simply tag-SNP or "synthetic association" markers, or if they are more likely to be true functional variants. The aforementioned two GWASs were both conducted in people of European descent; now, we have performed the first replication study in a Chinese Han population. In the original study, combined analysis demonstrated a strong association between alleles associated with shorter LTL and higher risk of CHD, but individually the lead SNPs at each of the telomere length-associated loci were not significantly associated with risk of CHD. However, among these loci, rs7675998 on 4q32.2 (NAF1) and rs9420907 chromosome $10 \mathrm{q} 24.33$ (OBFC1) showed the highest risks (1.43 and 0.41, respectively). Our study reconfirmed the association between rs7675998 and the disease, but no polymorphism was found for rs9420907 in the Chinese Han population. Moreover, in a subgroup analysis, rs 7675998 was also found to be related

Table 3 Effective allele ORs and adjusted ORs

\begin{tabular}{llllll}
\hline & Gene & $\begin{array}{l}\text { Effective } \\
\text { allele }\end{array}$ & $\begin{array}{l}\text { Other } \\
\text { allele }\end{array}$ & OR (95\% CI) & Adjusted OR (95\%) \\
\hline rs10936599 & TERC & T & C & $0.907(0.825-0.995)$ & $0.942(0.853-0.997)$ \\
rs2736100 & TERT & G & T & $1.013(0.925-1.109)$ & $1.003(0.920-1.007)$ \\
rs7675998 & NAFI & A & G & $2.104(1.887-2.345)$ & $1.913(1.702-2.121)$ \\
rs9420907 & OBFCI & A & C & N/A & N/A \\
rs8105767 & ZNF208 & A & G & $0.965(0.874-1.065)$ & $0.982(0.901-1.016)$ \\
rs755017 & RTELI & A & G & $1.211(1.106-1.327)$ & $1.120(1.007-1.204)$ \\
rsIII25529 & ACYP2 & C & A & $1.033(0.943-1.131)$ & $1.008(0.93 I-1.073)$ \\
\hline
\end{tabular}

Abbreviations: $\mathrm{OR}$, odds ratio; $\mathrm{Cl}$, confidence interval; N/A, not available. 
Table 4 Mean onset age of CHD for each genotype of rs7675998

\begin{tabular}{clc}
\hline Genotype & $\begin{array}{l}\text { Age (years) } \\
\text { (mean } \pm \text { SD) }\end{array}$ & $P$ \\
\hline rs7675998 & & 0.012 \\
AA & $52.6 \pm 10.4$ & \\
AG & $54.2 \pm 11.3$ & \\
GG & $59.5 \pm 9.2$ & \\
\hline
\end{tabular}

Abbreviations: CHD, coronary heart disease; SD, standard deviation.

to the onset age of CHD, verifying the hypothesis that those individuals who have inherited and are born with shorter telomeres might be more prone to $\mathrm{CHD}$, and their onset age of CHD may also be determined by LTL.

In conclusion, in a Chinese Han population, NAF1 gene encoding proteins with known function in telomere biology may influence both the possibility and the onset age of CHD, as previously reported in European studies.

\section{Disclosure}

The authors have no conflicts of interest to declare.

\section{References}

1. Yusuf S, Hawken S, Ôunpuu S, et al. Effect of potentially modifiable risk factors associated with myocardial infarction in 52 countries (the INTERHEART study): case-control study. Lancet. 2004;364(9438):937-952.

2. Evans A, Van Baal GC, McCarron P, et al. The genetics of coronary heart disease: the contribution of twin studies. Twin Res. 2003;6(5): 432-441.

3. Kelpis TG, Anastasiadis K, Nimatoudis I, Kelpi MG, Hadjimiltiades S, Papakonstantinou C. Prevalence of "distressed" personality in patients with coronary artery disease and its correlation with morbidity after coronary surgery. Hellenic J Cardiol. 2013;54(5):362-367.

4. Brouilette SW, Moore JS, McMahon AD, et al. Telomere length, risk of coronary heart disease, and statin treatment in the West of Scotland Primary Prevention Study: a nested case-control study. Lancet. 2007; 369(9556):107-114.

5. Farzaneh-Far R, Cawthon RM, Na B, Browner WS, Schiller NB, Whooley MA. Prognostic value of leukocyte telomere length in patients with stable coronary artery disease: data from the heart and soul study. Arterioscler Thromb Vasc Biol. 2008;28(7):1379-1384.

6. Brouilette SW, Whittaker A, Stevens SE, van der Harst P, Goodall AH, Samani NJ. Telomere length is shorter in healthy offspring of subjects with coronary artery disease: support for the telomere hypothesis. Heart. 2008;94(4):422-425.
7. Njajou OT, Cawthon RM, Damcott CM, et al. Telomere length is paternally inherited and is associated with parental lifespan. Proc Natl Acad Sci U S A. 2007;104(29):12135-12139.

8. Hodes RJ. Telomere length, aging, and somatic cell turnover. $J$ Exp Med. 1999;190(2):153-156.

9. Benetos A, Okuda K, Lajemi M, et al. Telomere length as an indicator of biological aging: the gender effect and relation with pulse pressure and pulse wave velocity. Hypertension. 2001;37(2 Pt 2):381-385.

10. Blasco MA. Telomere length, stem cells and aging. Nat Chem Biol. 2007;3(10):640-649.

11. Codd V, Nelson CP, Albrecht E, et al. Identification of seven loci affecting mean telomere length and their association with disease. Nat Genet. 2013;45(4):422-427.

12. Wong JM, Collins K. Telomere maintenance and disease. Lancet. 2003;362(9388):983-988.

13. Blackburn EH. Telomere states and cell fates. Nature. 2000; 408(6808): 53-56.

14. Artandi SE. Telomere shortening and cell fates in mouse models of neoplasia. Trends Mol Med. 2002;8(1):44-47.

15. Harris SE, Martin-Ruiz C, von Zglinicki T, Starr JM, Deary IJ. Telomere length and aging biomarkers in 70-year-olds: the Lothian Birth Cohort 1936. Neurobiol Aging. 2012;33(7):1486. e3-e8.

16. Salvioli S, Monti D, Lanzarini C, et al. Immune system, cell senescence, aging and longevity - inflamm-aging reappraised. Curr Pharm Des. 2013;19(9):1675-1679.

17. Effros RB. T cell replicative senescence: pleiotropic effects on human aging. Ann N Y Acad Sci. 2004;1019:123-126.

18. Finch CE, Crimmins EM. Inflammatory exposure and historical changes in human life-spans. Science. 2004;305(5691):1736-1739.

19. Heitzer T, Schlinzig T, Krohn K, Meinertz T, Münzel T. Endothelial dysfunction, oxidative stress, and risk of cardiovascular events in patients with coronary artery disease. Circulation. 2001;104(22): 2673-2678.

20. Harrison D, Griendling KK, Landmesser U, Hornig B, Drexler H. Role of oxidative stress in atherosclerosis. Am J Cardiol. 2003;91(3A): $7 \mathrm{~A}-11 \mathrm{~A}$.

21. von Zglinicki T. Oxidative stress shortens telomeres. Trends Biochem Sci. 2002;27(7):339-344.

22. Sampson MJ, Winterbone MS, Hughes JC, Dozio N, Hughes DA. Monocyte telomere shortening and oxidative DNA damage in type 2 diabetes. Diabetes Care. 2006;29(2):283-289.

23. Adaikalakoteswari A, Balasubramanyam M, Mohan V. Telomere shortening occurs in Asian Indian Type 2 diabetic patients. Diabet Med. 2005;22(9):1151-1156.

24. Valdes AM, Andrew T, Gardner JP, et al. Obesity, cigarette smoking, and telomere length in women. Lancet. 2005;366(9486):662-664.

25. Virtanen M, Nyberg ST, Batty GD, et al; IPD-Work Consortium. Perceived job insecurity as a risk factor for incident coronary heart disease: systematic review and meta-analysis. BMJ. 2013;347:f4746.

26. Epel ES, Blackburn EH, Lin J, et al. Accelerated telomere shortening in response to life stress. Proc Natl Acad Sci U S A. 2004;101(49): 17312-17315.
Clinical Interventions in Aging

\section{Publish your work in this journal}

Clinical Interventions in Aging is an international, peer-reviewed journal focusing on evidence-based reports on the value or lack thereof of treatments intended to prevent or delay the onset of maladaptive correlates of aging in human beings. This journal is indexed on PubMed Central, MedLine, the American Chemical Society's 'Chemical Abstracts

\section{Dovepress}

Service' (CAS), Scopus and the Elsevier Bibliographic databases. The manuscript management system is completely online and includes a very quick and fair peer-review system, which is all easy to use. Visit http://www.dovepress.com/testimonials.php to read real quotes from published authors. 